\title{
Treatment-Resistant Actinomycosis Reveals Concomitant Systemic Vasculitis
}

Faris Althubaiti MD, MAS-CR ${ }^{*}$, Sylvain Godreuil $\mathrm{MD}^{2}, \mathrm{PhD}$, Muriel Lalande MD, MSc ${ }^{3}$, Aurelia Carbasse $\mathrm{MD}^{4}$, Yassine al Tabaa MD, $\mathrm{PhD}^{5}$, Hélène Vernhet $\mathrm{MD}, \mathrm{PhD}^{6}$, Vanessa Szableski, $\mathrm{MD}^{7}$, Valerie Costes $\mathrm{MD} \mathrm{PhD}^{8}$, Eric Jeziorski MD, $\mathrm{PhD}^{9}$

\footnotetext{
${ }^{1}$ Université de Montpellier, Hôpital Arnaud de Villeneuve, Département urgences post-urgences. 371, Avenue du Doyen Gaston Giraud, 34295 Montpellier Cedex 5, France, King Abdulaziz University, Departement of Pediatrics, Jeddah, Saudi Arabia

${ }^{2}$ Service de Bactériologie, CHU Montpelier, Montpellier, France, Université de Montpellier UMR MIVEGEC, UMR IRD 224-CNRS, Montpellier, France

${ }^{3,4}$ Hôpital Arnaud de Villeneuve, Département urgences post-urgences. 371, Avenue du Doyen Gaston Giraud, 34295 Montpellier Cedex 5, France

5Departement D'imagerie Nucléaire, CHU Montpellier, Montpellier, France

${ }^{6}$ Departement d'imagerie, CHU Montpellier, Montpellier, France

${ }^{7}$ Département de Biopathologie Cellulaire et Tissulaire des Tumeurs, CHU Montpellier

${ }^{8}$ Département de Biopathologie Cellulaire et Tissulaire des Tumeurs, CHU Montpellier, Pathogenesis and Control of Chronic Infections, INSERM, Universite de Montpellier,

${ }^{9}$ Centre Hospitalier Universitaire Montpellier, Hôpital Arnaud de Villeneuve, 371, Avenue du Doyen Gaston Giraud, 34295 Montpellier Cedex 5, France, Pathogenesis and Control of Chronic Infections, INSERM, Universite de Montpellier, Montpellier, France
}

Takayasu Arteritis (TA) is a granulomatous systemic vasculitis that mainly involves the aorta and its large branches. Its etiology is unknown; however, several triggering factors have been reported. Infectious agents are thought to have a role in the disease pathogenesis mainly mycobacterium tuberculosis. Early diagnosis is sometimes challenging in the absence of adequately sensitive biomarkers and the intermittent nature of this disease. We present here a thirteen-yearold girl who fell down on her chin and fractured her $21^{\text {st }}$ tooth, presented to us with posttraumatic febrile illness and unilateral headaches. A diagnosis of posttraumatic left mandibular osteitis was done initially supported by clinicoradiological and histopathological findings. Due to treatment resistance and appearance of other clinical features mainly "Arterial hypertension and prominent carotid pulsations" the diagnosis of TA was considered. Treatment by corticotherapy was initiated but because of corticodependence, infliximab and methotrexate were introduced and permitted us to stop steroid therapy, without any flare or new attempt 4 years after diagnosis. We suggest that these rare granulomatous diseases can co-occur and should be evoked in atypically evolving cases.

Keywords: Granulomatous disease, systemic vasculitis, Takayasu disease, Mandibular actinomycosis.

Copyright @ 2020: This is an open-access article distributed under the terms of the Creative Commons Attribution license which permits unrestricted use, distribution, and reproduction in any medium for non-commercial use (NonCommercial, or CC-BY-NC) provided the original author and source are credited.

\section{LIST OF ABBREVIATIONS:}

\section{TA: Takayasu Arteritis}

PReS: Pediatric Rheumatology European Society

EULAR: European League Against Rheumatism

\section{INTRODUCTION}

Actinomyces are gram-positive, non-acid-fast, anaerobic to microaerobic filamentous organisms that cause chronic granulomatous disease. They are commensals of the oropharynx and the gastrointestinal and genitourinary tracts. Actinomycosis is characterized by a wide spectrum of clinical presentations including constitutional symptoms. Takayasu arteritis (TA) is a rare granulomatous systemic vasculitis that mainly involves the aorta and its large branches. Its etiology is unknown; however, several triggering factors have been reported in literature, including infectious agents, mainly Mycobacterium tuberculosis. Genetic susceptibility and inflammatory diseases are also reported to play a role in disease pathogenesis. Here, we present a 13-year-old patient who demonstrated a challenging diagnostic course. After year-long antibiotic treatment for actinomycosis following posttraumatic dental infection provided only partial relief for this patient, that revealed an underlying systemic issue of TA diagnosed with magnetic resonance angiography. The co-occurrence of TA and actinomycosis has not previously been reported. We 
suggest that there might be an association between Actinomyces and Takayasu arteritis.

\section{Case Presentation}

A 13-year-old girl of Moroccan origin presented initially with a traumatic dental fracture of tooth 21. Local treatment with removal of dental braces was done by her dentist. One month later, she reported again with a febrile illness and a unilateral cervical mass associated with unilateral headaches. Clinically, she was febrile with left mandibular swelling and cervical lymphadenopathy. A course of josamycine was prescribed with no clinical improvement.

At the time of referral, she presented with persistence of her initial symptoms, an appearance of fatigue, and an increase in mandibular mass size in association with mandibular pain and tenderness. A blood workup revealed an inflammatory syndrome with C-reactive protein (CRP) at $82 \mathrm{mg} / \mathrm{L}$, anemia with hemoglobin levels at $9.2 \mathrm{~g} / \mathrm{dL}$ and a mean corpuscular volume of $67 \mathrm{fL} /$ cell, and ferritinemia at $26 \mathrm{ug} / \mathrm{L}$. Panoramic dental X-ray images were normal. A computed tomography scan and magnetic resonance imaging of her head and neck revealed myositis of the left masseter and medial pterygoid muscles with submandibular adenopathy and osteitis by contiguity (Figure-1).

A course of oral amoxicillin and clavulanic acid was prescribed without much improvement, and iron supplementation was prescribed to address the iron deficiency. Ultrasound-guided and protected muscular and bone biopsies were performed. Tissue culture was positive for penicillin-sensitive Actinomyces with a minimum inhibitory concentration of $190 \mathrm{mg} / \mathrm{L}$. The initial histopathology reports a granulomatous vasculitis associated with micro-emboli of the masseter muscle that could be attributed to local infection of either tuberculosis or actinomycosis, or to systemic vasculitis (Figure-2). A diagnosis of mandibular actinomycosis was retained because of the clinical presentation and the positivity of the protected samples.

She was treated with first-line antibiotics, with intravenous amoxicillin (200 $\mathrm{mg} / \mathrm{kg} /$ day $)$ plus clindamycin (45 mg/kg/day) for one month, followed by oral therapy with the same antibiotics and dosage for 3 months. Due to the persistence of mild inflammatory signs in the masseter associated with an elevated CRP (66.9 $\mathrm{mg} / \mathrm{L})$, intravenous antibiotic therapy was then prescribed with amoxicillin plus clavulanic acid (co- amoxiclav) for 5 months, followed by oral amoxicillin plus clavulanic acid and clindamycin for 3 months. During this treatment, the nadir of CRP was $16 \mathrm{mg} / \mathrm{L}$ and varied between 30 and $60 \mathrm{mg} / \mathrm{L}$ in association with transient recurrence of left submandibular induration.

At the end of this sequence, the reappearance of headaches, in addition to the development of hypertension, bilateral carotid murmur, and right femoral thrill led us to perform vascular ultrasound and magnetic resonance angiography. Magnetic resonance angiography showed left common carotid arteritis, diffuse sub-diaphragmatic aortitis, supra and subrenal aneurysms, and stenosis of the right and left common iliac arteries (Figure-3). A diagnosis of Takayasu's arteritis was made based on the clinical course and radiological findings that complete the European League Against Rheumatism (EULAR) diagnostic criteria. Antibiotic therapy was stopped, and she was then treated with prednisone $(2 \mathrm{mg} / \mathrm{kg}$ for 1 month with a progressive decrease), with a good response for mandibular lesions, hypertension, and inflammatory markers, but persistence of multiple stenosis. Because of corticodependence, infliximab and methotrexate were introduced and permitted us to stop steroid therapy, without any flare or new attempt 4 years after diagnosis.

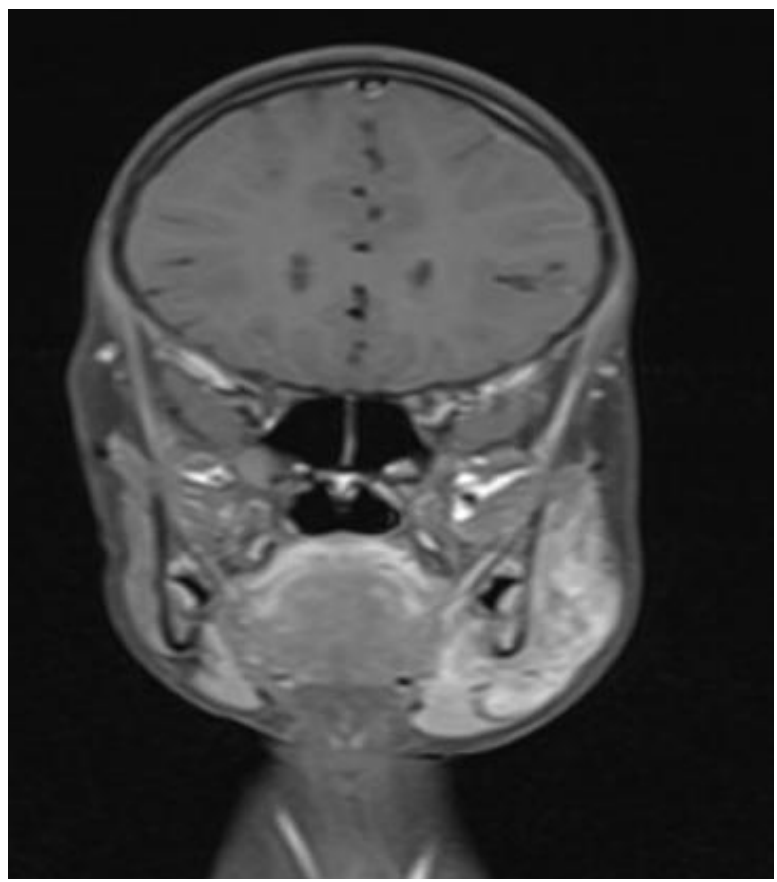

Fig-1: Magnetic resonance imaging of the head and neck shows a left submandibular mass 


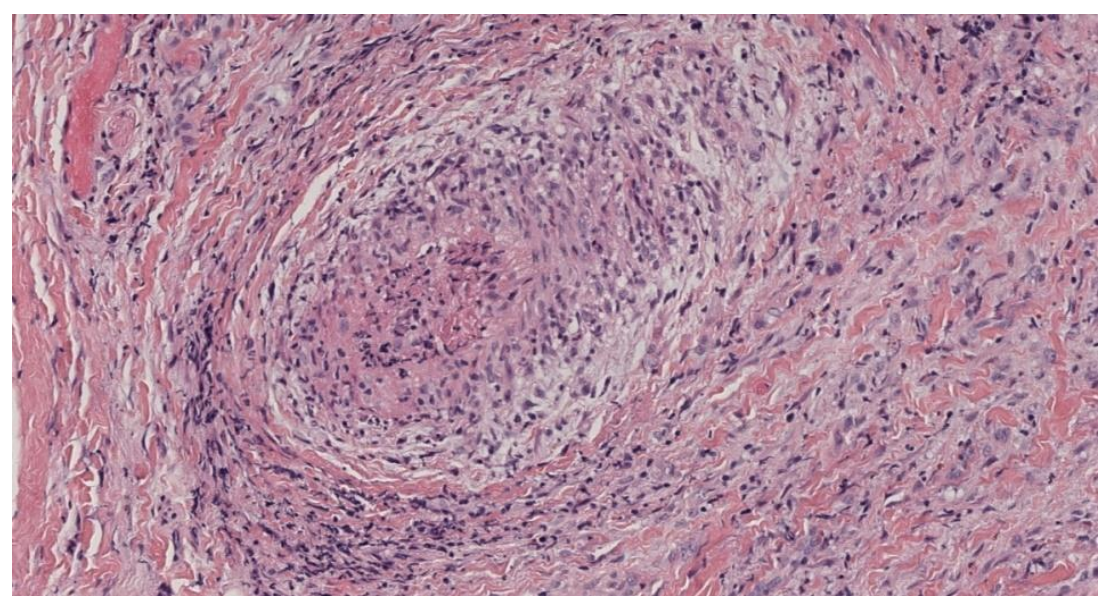

Fig-2: Photos of vascular sections showing parietal wall inflammatory infiltration with histocyte predominance. Stained with hematoxylin and eosin, magnification $\times 20$

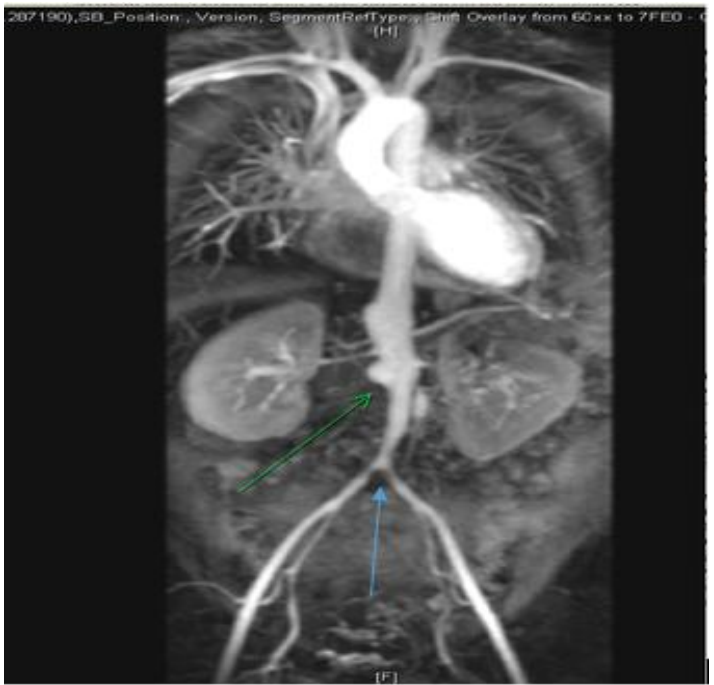

Fig-3: Magnetic resonance angiography with the green arrow pointing to supra- and subrenal aneurysms, and blue arrow pointing femoral stenosis

\section{DISCUSSION}

Actinomycosis is not a common infection, affecting mainly immunocompromised persons. The cervicofacial type is the most common, which can arise following dental manipulation or a traumatic event to the mouth and face. This kind of infection can also occur spontaneously in patients with poor dental hygiene. Fevers and chronic painless or painful soft tissue swelling of the perimandibular region with lymphadenopathy at late stages are the most commonly presenting symptoms. Local tissue involvement, such as involvement of bone and muscle (periostitis and osteomyelitis), can occur in up to $11.7 \%$ of cases, with the mandible being the most common site of bone disease $[1,2]$.

Isolation of Actinomyces is difficult, especially after the use of antibiotics, with a failure rate up to more than $50 \%$. Gram staining is a more sensitive technique, but molecular methods are more rapid and accurate for identification. Diagnostics are challenging because Actinomycetes belong to the commensal flora of the mouth. Antibiotic therapy for actinomycosis should encompass a broad spectrum that covers other organisms depending on the site of infection. $\beta$-lactam and $\beta$-lactamase inhibitors are traditionally recommended for a duration ranging from six to twelve months depending on the severity of disease and whether surgical resection was involved. In our case, the clinical presentation, positive protected samples, and initial evolution validate the initial diagnosis.

The diagnosis of TA was certain because of the presentation and initial histology that identified vasculitis that was consistent with systemic vasculitis. This is a rare granulomatous systemic vasculitis that mainly involves the aorta and its large branches, leading to stenosis and aneurysm formation in severe cases. It affects all ethnicities with a higher incidence in Southeast Asia, Africa, and South and Central America $[3,4]$. It has an estimated incidence of 2.6/1000000 over all age groups with a female predominance $[5,6]$. There is a broad spectrum of clinical presentations, ranging from constitutional symptoms in the early acute inflammatory phase to vascular occlusive disease manifestations in the chronic phase. Consensus diagnostic criteria have been suggested by the Pediatric Rheumatology European Society (PReS) and the European League Against Rheumatism (EULAR) that mandate the presence of a radiographic abnormality in association with at least one of the other TA features [7]. The clinical presentation of our patient is compatible with those described in large case series, such as Brunner et al., [6] that have done a systematic review of 241 patients and reported that hypertension $(82.6 \%)$, headache $(31.4 \%)$, fever $(29.4 \%)$, dyspnea $(23.3 \%)$, weight loss $(22.1 \%)$, and bruits $(16.5 \%)$ were the most common clinical features at the time of diagnosis in children.

The etiological and predisposing factors for TA are not clearly identified. Genetic susceptibility influenced by environmental and infectious agents is strongly suggested [8]. TA can also occur in association with a variety of autoimmune diseases like systemic 
lupus erythematosus and inflammatory bowel disease [9]. Heat shock protein-65 expression in vascular tissue, which is also produced by other tissues in response to stress, is thought to have an important role in the pathogenesis of TA [10]. Mycobacteria is the most commonly suspected infectious agent to cause TA. The granulomatous nature of the disease, as well as molecular similarity between the Mycobacterium heat shock protein and its human homologue (hHSP60) prompting an immune response, are the most apparent links $[11,12]$. To our knowledge, our case is the first to report the co-occurrence of TA and actinomycosis. The similarity between Mycobacteria and Actinomyces in presenting a granulomatous reaction in tissue might provide a common etiologic mechanism related to TA, although there is currently no evidence demonstrating the nature of the link between tuberculosis and TA.

There are several reasons why TA was not initially considered in the diagnosis of the present case. Orofacial actinomycosis could require a long course of systemic antibiotics in the case of osteitis in an immunocompetent patient. For our patient, after remission, when the second-line therapeutic window was completed, moderate systemic inflammatory features persisted but neither suppurative inflammation nor abscess or sinus formation occurred. Furthermore, the imaging features are usually nonspecific in a diagnosis of actinomycosis, as they were for our patient, as they might be similar to those found in many other inflammatory or neoplastic pathologies $[13,14]$.

An early onset of TA at the time of presentation is highly suspected in this patient, but because of the mandibular trauma and the identification of Actinomyces in tissue culture, we could not rule out concomitant actinomycosis. We conclude that our patient contracted both diseases; the rarity of them and the TA physiopathologic hypothesis suggest a relationship, but it is impossible to assert which disease has promoted the other.

\section{Conclusion}

We emphasize here the importance of considering vasculitis in the list of differential diagnoses of infectious cases that do not show a good response to antibiotics therapy. A negative initial workup does not rule out vasculitis and it is always worth it to repeat the work up as the disease progresses.

\section{REFERENCES}

1. Oostman O, Smego RA. Cervicofacial Actinomycosis: Diagnosis and Management. Curr Infect Dis Rep. 2005;7(3):170-174.
2. Schaal KP, Lee HJ. Actinomycete infections in humans--a review. Gene. 1992;115(1-2):201-211. doi:10.1016/0378-1119(92)90560-c

3. Weiss PF. Pediatric vasculitis. Pediatr Clin North Am. doi:10.1016/j.pcl.2012.03.013

4. Richards BL, March L, Gabriel SE. Epidemiology of large-vessel vasculidities. Best Pract Res Clin Rheumatol. doi:10.1016/j.berh.2010.10.008

5. Kerr GS, Hallahan CW, Giordano J, Leavitt RY, Fauci AS, Rottem M, Hoffman GS. Takayasu arteritis. Annals of internal medicine. 1994 Jun 1;120(11):919-29.

6. Brunner J, Feldman BM, Tyrrell PN, KuemmerleDeschner JB, Zimmerhackl LB, Gassner I, Benseler SM. Takayasu arteritis in children and adolescents. Rheumatology. 2010 Oct 1;49(10):1806-14.

7. Ozen S, Ruperto N, Dillon MJ, Bagga A, Barron K, Davin JC, Kawasaki T, Lindsley C, Petty RE, Prieur AM, Ravelli A. EULAR/PReS endorsed consensus criteria for the classification of childhood vasculitides. Annals of the rheumatic diseases. 2006 Jul 1;65(7):936-41.

8. Terao C. Revisited HLA and non-HLA genetics of Takayasu arteritis--where are we? J Hum Genet. 2016;61(1):27-32. doi:10.1038/jhg.2015.87

9. 9. Al Abrawi S, Fouillet-Desjonqueres $\mathrm{M}$, David L, Barral X, Cochat P, Cimaz R. Takayasu arteritis in children. Pediatr Rheumatol Online J. 2008;6:17. doi:10.1186/1546-0096-6-17

10. Arnaud L, Haroche J, Mathian A, Gorochov G, Amoura Z. Pathogenesis of Takayasu's arteritis: a 2011 update. Autoimmun Rev. 2011;11(1):61-67. doi:10.1016/j.autrev.2011.08.001

11. Aggarwal A, Chag M, Sinha N, Naik S. Takayasu's arteritis: role of Mycobacterium tuberculosis and its $65 \mathrm{kDa}$ heat shock protein. Int J Cardiol. 1996;55(1):49-55. doi:10.1016/01675273(96)02660-5

12. Kumar Chauhan S, Kumar Tripathy N, Sinha N, Singh M, Nityanand S. Cellular and humoral immune responses to mycobacterial heat shock protein-65 and its human homologue in Takayasu's arteritis. Clin Exp Immunol. 2004;138(3):547-553. doi:10.1111/j.13652249.2004.02644.x

13. Kim TS, Han J, Koh WJ, Choi JC, Chung MJ, Lee JH, Shim SS, Chong S. Thoracic actinomycosis: CT features with histopathologic correlation. American Journal of Roentgenology. 2006 Jan;186(1):225-31.

14. Pickhardt PJ, Bhalla S. Unusual nonneoplastic peritoneal and subperitoneal conditions: CT findings. Radiographics. 2005;25(3):719-730. doi:10.1148/rg.253045145 